\section{Moving S1P into osteoporosis}

\section{By Brian Moy, Staff Writer}

Researchers at the NIH have found that agonizing sphingosine 1phosphate receptor 1 regulates bone homeostasis and the migration of osteoclast precursors. The discovery adds osteoporosis to the list of potential indications for companies working with the sphingolipid signaling pathway, which is already being targeted in cancer and autoimmune diseases such as multiple sclerosis (MS) and was recently reported to play a role in decreasing cytokine-related pulmonary tissue damage during influenza infection.

Osteoporosis is mediated by the balance between bone-resorbing osteoclasts and bone-forming osteoblasts. ${ }^{1}$ In a paper in Nature, the NIH team reported that sphingosine 1-phosphate (S1P) induced chemotaxis and controlled the migration of sphingosine 1-phosphate receptor 1 (S1PR1)-expressing osteoclast precursor cells in vitro. Mice with S1PR1deficient osteoclasts showed a greater number of mature osteoclasts attached to the bone surface and had significantly lower bone density measurements than wild-type littermate controls $(p=0.004)$.

In wild-type mice with ovariectomy-induced osteoporosis, the S1PR1 agonist FTY720 decreased the number of mature osteoclasts attached to the bone surface and prevented bone density loss compared with what was seen using vehicle control. ${ }^{2}$

Novartis AG's Fingolimod (FTY720) is in Phase III testing to treat relapsing-remitting MS (RRMS). Novartis has rights to FTY720 from Mitsubishi Tanabe Pharma Corp.

"Our findings identify a critical receptor that controls osteoclastogenesis that can be targeted to reduce the number of mature osteoclasts attached to bone and ultimately provide relief of osteoporosis," said Ronald Germain, the principal investigator of the study and deputy chief of the laboratory of immunology and chief of the lymphocyte biology section at the National Institute of Allergy and Infectious Diseases (NIAID). Germain told SciBX the findings are neither patented nor licensed.

Although agonizing S1PR1 did not increase bone formation, Gerard Karsenty, chair of the Department of Genetics and Development at Columbia University Medical Center, said the NIAID findings "certainly explore a novel way to block bone resorption and treat osteoporosis."

He suggested that the NIH researchers test their findings in other animal models of disease and compare the results with those of marketed osteoporosis drugs.

Along a similar line, Germain told $S c i B X$ it would be useful to analyze bone density measurements in MS patients who have been treated with FTY720.

However, FTY720 itself may not be an optimal osteoporosis candidate. Germain cautioned that because the compound is an immunosuppressant, "we have to come up with a strategy or drug formulation that can regulate the migratory behavior of osteoclast precursors without suppressing lymphocytes."

Roger Sabbadini, VP and CSO of Lpath Inc., had similar thinking. "Although treatment with FTY720 is likely showing efficacy in this animal model of osteoporosis by a mechanism involving the downregulation of several S1PRs," he said, further studies should investigate the exact mechanism behind how FTY720 lowers the number of mature osteoclasts in order to identify other ways to modulate the S1PR1 signaling system.

Finding other modulators is important, he said, because the Nature paper "quite convincingly suggests that intervening in the S1P pathway could be useful in the treatment of osteoporosis."

Lpath's Asonep, a mAb against S1P, is in Phase I testing to treat cancer. But before contemplating osteoporosis as a clinical indication, Sabbadini said, the company would have to test its $\mathrm{mAb}$ in preclinical models of the disease. Asonep is a systemic version of sonepcizumab, which in turn is a humanized version of Lpath's Sphingomab, a murine antibody.

Merck Serono S.A., a division of Merck KGaA, has an exclusive worldwide license to Asonep. Lpath retains rights to iSonep, an ocular formulation of sonepcizumab that is in Phase I testing for wet age-related macular degeneration (AMD).

The other S1PR1 agonist in the clinic is R3477 from Actelion Ltd. and Roche. The compound is in Phase I testing to treat multiple autoimmune diseases.

In addition to osteoporosis, companies in the S1PR1 space recently had another potential indication land in their laps. Last month, researchers at The Scripps Research Institute reported in the Proceedings of the National Academy of Sciences that a single low dose of an S1PR1-binding sphingosine analog administered into the lungs of mice infected with influenza substantially lowered the release of cytokines and chemokines from dying, virus-infected cells. ${ }^{3}$

Moy, B. SciBX 2(8); doi:10.1038/scibx.2009.302

Published online Feb. 26, 2009

\section{REFERENCES}

1. Teitelbaum, S.L. Science 289, 1504-1508 (2000)

2. Ishii, M. et al. Nature; published online Feb. 8, 2009; doi:10.1038/nature07713

Contact: Ronald N. Germain, National Institute of Allergy and Infectious Diseases, Bethesda, Md. e-mail: rgermain@niaid.nih.gov

3. Marsolais, D. et al. Proc. Natl. Acad. Sci. USA; published online Jan. 19, 2009; doi:10.1073/pnas.0812689106

Contact: Michael B.A. Oldstone, The Scripps Research Institute, La Jolla, Calif.

e-mail: mbaobo@scripps.edu

Contact: Hugh Rosen, same affiliation as above

e-mail: hrosen@scripps.edu 


\section{TARGETS \& MECHANISMS}

COMPANIES AND INSTITUTIONS MENTIONED

Actelion Ltd. (SIX:ATLN), Allschwil, Switzerland

Columbia University Medical Center, New York, N.Y.

Merck KGaA (Xetra:MRK), Darmstadt, Germany

Merck Serono S.A., Geneva Switzerland

Lpath Inc. (OTCBB:LPTN), San Diego, Calif.

Mitsubishi Tanabe Pharma Corp. (Tokyo:4508; Osaka:4508),
Osaka, Japan

National Institute of Allergy and Infectious Diseases,

Bethesda, Md.

National Institutes of Health, Bethesda, Md.

Novartis AG (NYSE:NVS; SIX:NOVN), Basel, Switzerland

Roche (SIX:ROG), Basel, Switzerland

The Scripps Research Institute, La Jolla, Calif. 